J. Domenech ${ }^{1}$

M. Wyers ${ }^{2}$

J.-P. Braun ${ }^{3}$

P. Formenty ${ }^{4}$

\title{
Le syndrome nerveux des ovins en Côte-d'Ivoire. I. Étude épidémiologique et clinique, méthodes de diagnostic et traitement
}

DOMENECH (J.), WYERS (M.), BRAUN (J.-P.), FORMENTY (P.). Le syndrome nerveux des ovins en Côte-d'Ivoire. I. Étude épidémiologique et clinique, méthodes de diagnostic et traitement. Revue Elev. Méd. vét. Pays trop., 1993, 46 (3) : 423-430

Le "syndrome nerveux des ovins" en Côte-d'Ivoire est assimilable à la "nécrose du cortex cérébral" (NCC) due à une carence en vitamine B1. Tous les symptômes classiques de la NCC ont été observés (ataxie locomotrice suivie de paralysies) et les lésions histologiques de polioencéphalomalacie ont été mises en évidence. En revanche, les circonstances d'apparition de la maladie sont très différentes dans les deux cas : si la NCC est une maladie des jeunes ruminants à l'engrais dans les pays développés, le syndrome nerveux des ovins se manifeste essentiellement, en Côte-d'Ivoire, durant la saison sèche, lorsque le pâturage devient sec et rare et que la complémentation alimentaire est insuffisante. La cause principale, relativement univoque, est donc représentée par une dégradation brutale de la valeur nutritionnelle de la ration alimentaire, mais l'étiopathogénie précise de la maladie reste inconnue. Dans un troupeau atteint, 10 à $30 \mathrm{p} .100$ des animaux peuvent être malades et 80 à 90 p. 100 d'entre eux vont mourir. Sur le plan du diagnostic, aucune épreuve biochimique classique n'est suffisamment spécifique pour être retenue pour une confirmation formelle du syndrome nerveux. Il faut cependant signaler que les CK (créatinine kinase) sont très régulièrement augmentées et que les ASAT (aspartate aminotransférase) sont élevées dans 75 p. 100 des cas. Le diagnostic formel reste donc basé, dans les conditions actuelles pratiques de terrain en Afrique, sur l'efficacité du traitement avec la vitamine B1 et, pour l'animal mort, sur l'analyse histologique du cerveau.

Mots clés : Ovin - Trouble du système nerveux - Nécrose du cortex cérébral - Poliencéphalomalacie - Épidémiologie - Diagnostic - Thérapeutique - Histologie - Carence en vitamine - Thiamine - Côte-d'Ivoire.

\section{INTRODUCTION}

Depuis 1978, une nouvelle maladie des ovins a été signalée en Côte-d'Ivoire $\left(13^{*}\right)$. Cette affection se traduit par des symptômes nerveux de type ataxique puis paralytique et atteint les troupeaux de moutons faisant l'objet d'améliorations génétiques et zootechniques $(1,13,20)$. Au fur et à mesure que l'élevage intensifié se dévelop-

1. OUA-IBAR, PO Box 30786, Nairobi, Kenya.

2. Laboratoire d'Anatomie pathologique, École nationale vétérinaire, Case Postale 3013, 44087 Nantes Cedex, France.

3. Laboratoire de Biochimie, École nationale vétérinaire, 23, Chemin des Capelles, 31076 Toulouse Cedex, France.

4. Laboratoire central de Pathologie animale, B.P. 206, Bingerville. Côte- d'Ivoire.

Reçu le 17.7.1992, accepté le 16.9.1992.

* Les références bibliographiques se trouvent à la fin de la deuxième partie de cet article, p. 519 de ce volume. pait, le nombre de foyers augmentait, en particulier dans la moitié sud du pays, de climat humide et sub-humide.

L'origine nutritionnelle de cette maladie, dénommée dès le début "syndrome nerveux des ovins", a tout de suite été évoquée $(6,13)$ mais l'étiologie précise n'a pu être mise en évidence rapidement. Parmi les différentes hypothèses, l'intervention de la carence en vitamine B1 a été envisagée car les symptômes étaient en tous points comparables à ceux de la nécrose du cortex cérébral $(2$, $4,7,9,10,14,18,21,22$ ). Mais les données épidémiologiques divergent nettement de celles observées dans les pays développés. De plus, les lésions histologiques et l'efficacité du traitement avec la thiamine n'avaient pas été clairement mises en évidence avant l'année 1990 (8).

Les études menées en Côte-d'Ivoire entre 1989 et 1991 sur ce syndrome sont exposées en deux parties : la première fait état des données épidémiologiques et cliniques et des méthodes de diagnostic et de traitement. La deuxième partie abordera le problème de l'importance économique d'une maladie qui semble n'être pas limitée à la Côte-d'Ivoire puisqu'une affection très voisine, sinon identique, a été signalée dans d'autres pays africains, de climat et végétation similaires, comme lo Ghana(3) ou différents, comme le Sénégal (17).

Dans cette même partie, les résultats d'essais de prophylaxie et de calcul des coûts-bénéfices des plans de lutte seront présentés.

\section{MATÉRIEL ET MÉTHODES}

\section{Types d'élevage}

La maladie n'est signalée que dans des troupeaux de moutons de race Djallonké, élevés en unités de plusieurs dizaines à plusieurs centaines de têtes, et faisant l'objet d'efforts pour augmenter la productivité. La répartition géographique est essentiellement limitée à la moitié sud du pays, de climat humide et sub-humide (carte 1).

L'encadrement technique par la SODEPRA (Société de Développement des Productions animales), dont bénéficient ces troupeaux, suppose l'adhésion des éleveurs à toute une série de thèmes améliorateurs : conduite en troupeau, interdiction de la divagation, utilisation d'un parc de nuit, complémentation alimentaire en saison sèche, apport de sels minéraux, prophylaxies systématiques contre la peste des petits ruminants, la clavelée et les parasitoses gastro-intestinales et cutanées. 


\section{J. Domenech M. Wyers J.-P. Braun P. Formenty}

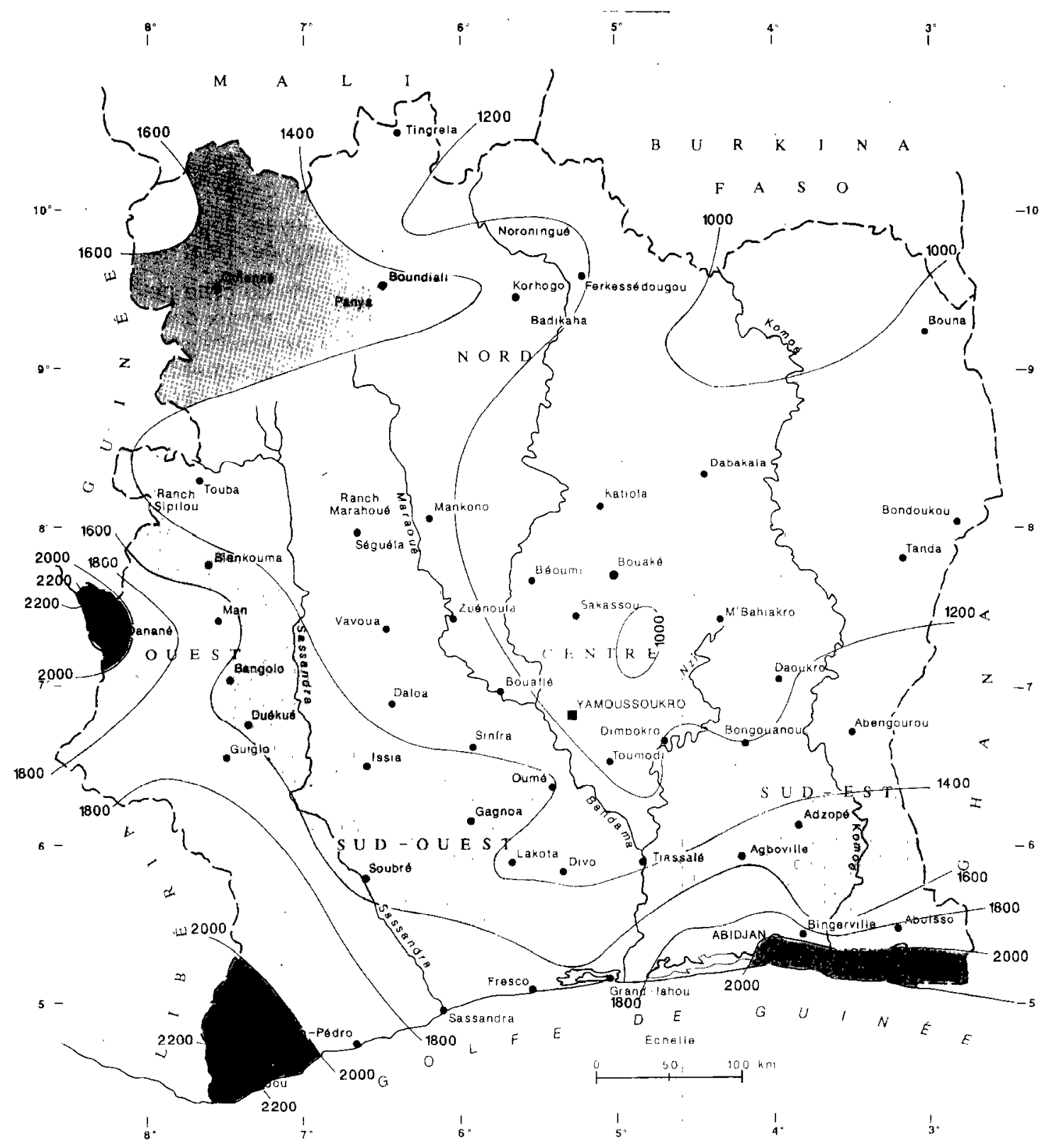

\section{LEGENDE}

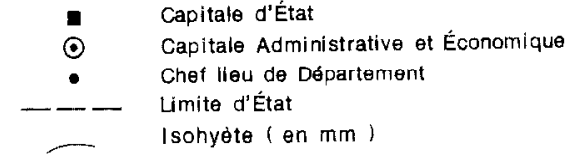




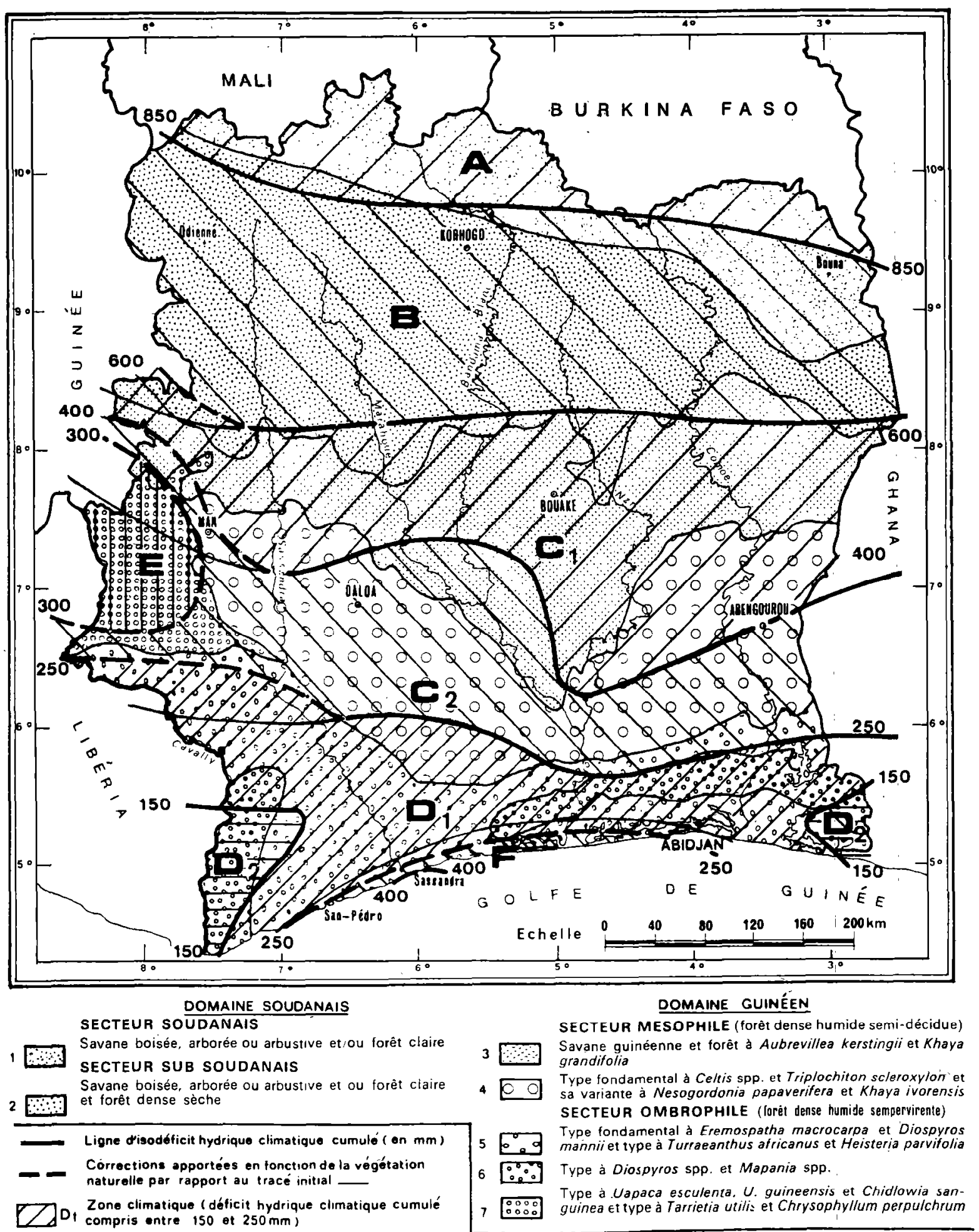


Le pâturage est à base de savane naturelle de zone guinéenne à soudanienne (carte 2) à laquelle s'ajoute ou se substitue, surtout dans le sud forestier, un pâturage artificiel de Panicum maximum.

\section{Prélèvements}

A l'occasion des visites des foyers, les interventions du laboratoire ont consisté en des examens épidémiologiques et cliniques, accompagnés de prélèvements sur l'animal vivant et en des autopsies d'animaux morts. Les prélèvements ont été effectués avec un matériel ordinaire, auquel s'ajoutait l'appareillage nécessaire à la centrifugation immédiate des sangs.

Les prélèvements de sang ont été recueillis, selon l'analyse à effectuer, en tube secs ou contenant des anticoagulants (EDTA, héparinate de lithium, fluorure de sodium oxalate de potassium). Pour le dosage de l'acide pyruvique, le sang a été déprotéinisé (acide perchlorique). Des fèces, pour recherches coproscopiques, et des frottis sanguins, pour diagnostic des hémoparasitoses, ont également été prélevées. Après autopsie, d'autres tissus (tissu nerveux, foie, rein...) ont été réfrigérés, congelés ou formolés, selon le cas, pour analyses bactériologiques, virologiques ou histologiques.

\section{Diagnostic de laboratoire}

Les analyses hématologiques ont porté sur l'hématocrite, la numération sanguine et la formule leucocytaire. Les dosages biochimiques sanguins ont concerné le sodium, le potassium, les chlorures, les bicarbonates, le calcium, le phosphore, le magnésium, les protéines totales, l'urée, la créatinine, les triglycérides, la phosphatase alcaline, les ALAT (alanine aminotranférase), les ASAT (aspartate aminotransférase), les GGT (gamma glutamyl transférase), la LDH (lactate déshydrogénase), les CK (créatinine kinase), l'acide lactique et l'acide pyruvique. Les méthodes classiques en usage ont été utilisées pour les analyses parasitologiques, bactériologiques et histologiques. Les recherches virologiques ont consisté en inoculations au souriceau nouveau-né et à l'oeuf embryonné.

\section{Traitement}

Les interventions ont porté sur 25 foyers (carte 3), à l'occasion desquelles 72 moutons ont été traités à des stades variés de la maladie. Ce traitement a reposé sur l'usage exclusif de vitamine B1 (chlorhydrate de thiamine), à la dose de $400 \mathrm{mg}$ par voie IV et/ou sous-cutanée, pendant 5 à 6 jours $(11,21,22)$. Le chlorhydrate de thiamine Hoffmann-Laroche en poudre a été remis en solution dans du sérum physiologique, à la concentration de $100 \mathrm{mg} / \mathrm{ml}$, et stérilisé, par filtration, au laboratoire. La conservation du produit étant présumée aléatoire

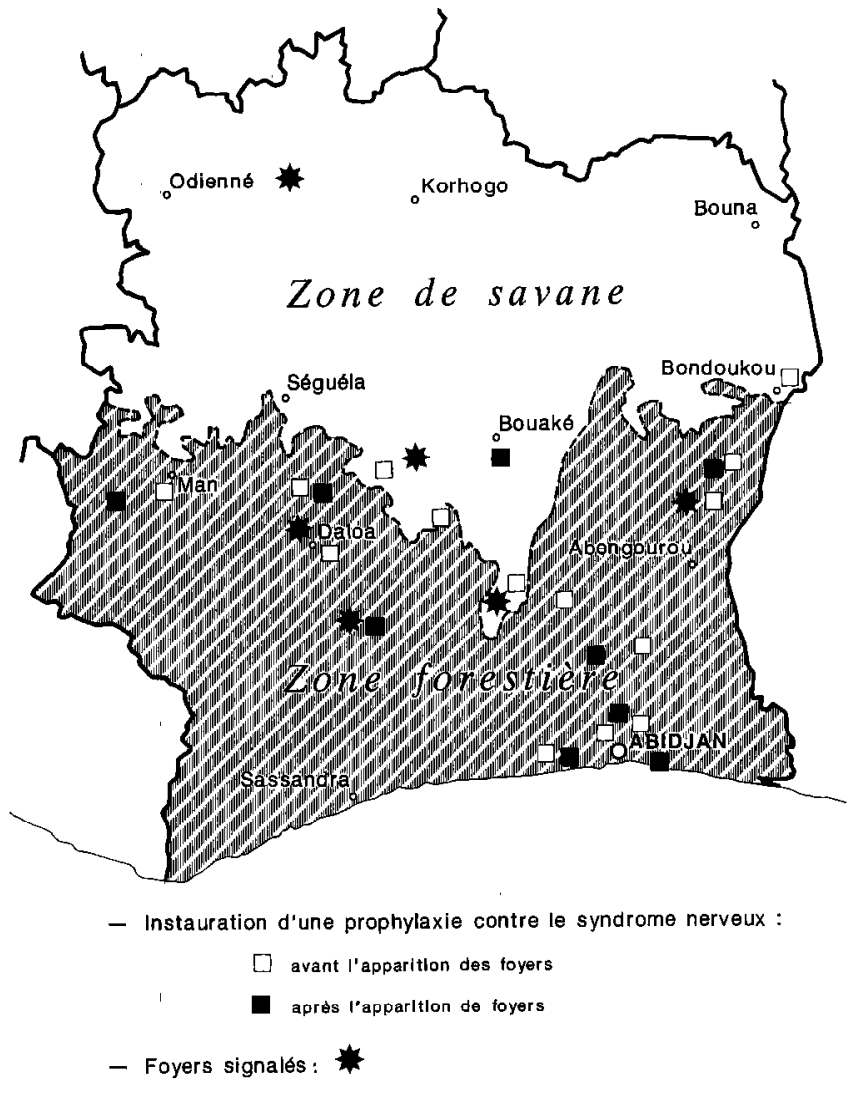

Carte 3 : Syndrome nerveux des ovins : foyers observés et essais de prophylaxie.

lorsqu'on n'est pas en milieu acide, la solution a donc été préparée un à quelques jours seulement avant son utilisation et conservée à $+4^{\circ} \mathrm{C}$.

\section{RÉSULTATS}

\section{Étude épidémiologique et clinique}

Dans la grande majorité des cas, la "nécrose du cortex cérébral" (NCC) apparaît dans des troupeaux de type amélioré, d'une dizaine à plusieurs centaines de têtes, durant la saison sèche, et dans la moitié sud du pays. Quelques exceptions existent cependant (1 foyer sur 27 observé dans le Nord). Lorsque le foyer apparaît, le pâturage, qu'il soit une savane naturelle ou un pâturage artificiel de Panicum maximum, est très dégradé et la complémentation alimentaire insuffisante ou inexistante. Les foyers évoluent sur 2 à 3 semaines. Le taux de morbidité atteint 10 à 30 p. 100 et, parmi les malades, 80 à 90 p.100 mourront si aucun traitement spécifique n'est instauré. La maladie touche surtout les adultes, très exceptionnellement les jeunes à la mamelle.

Le tableau clinique observé sur plus de 300 malades est dominé par des signes nerveux : ataxie locomotrice sui- 
Le syndrome nerveux des ovins en Côte-d'Ivoire. Étude épidémiologique et clinique

J. Domenech et coll.

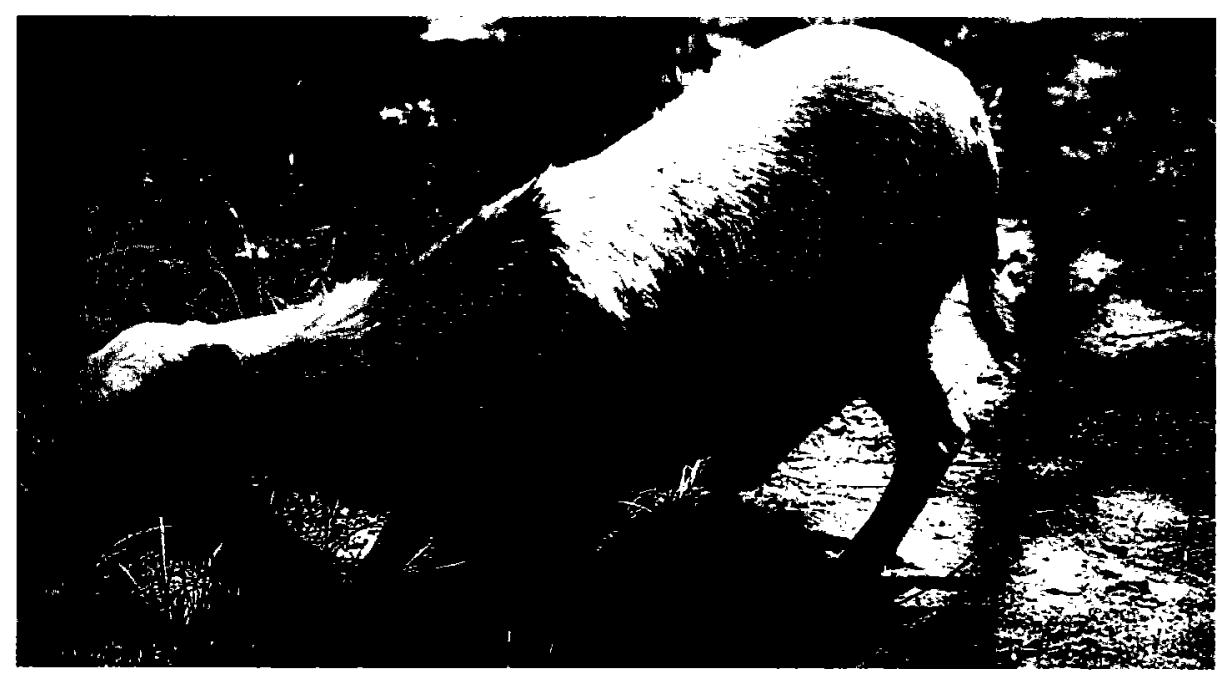

Début de la maladie

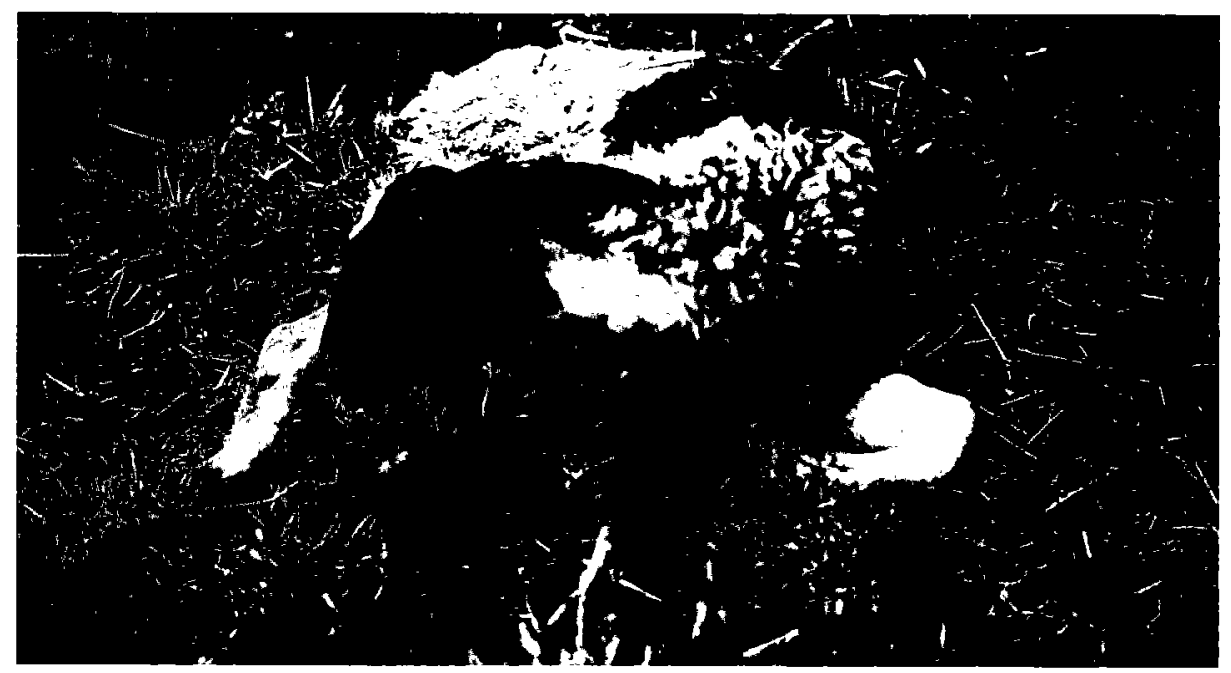

Début de la phase de décubitus

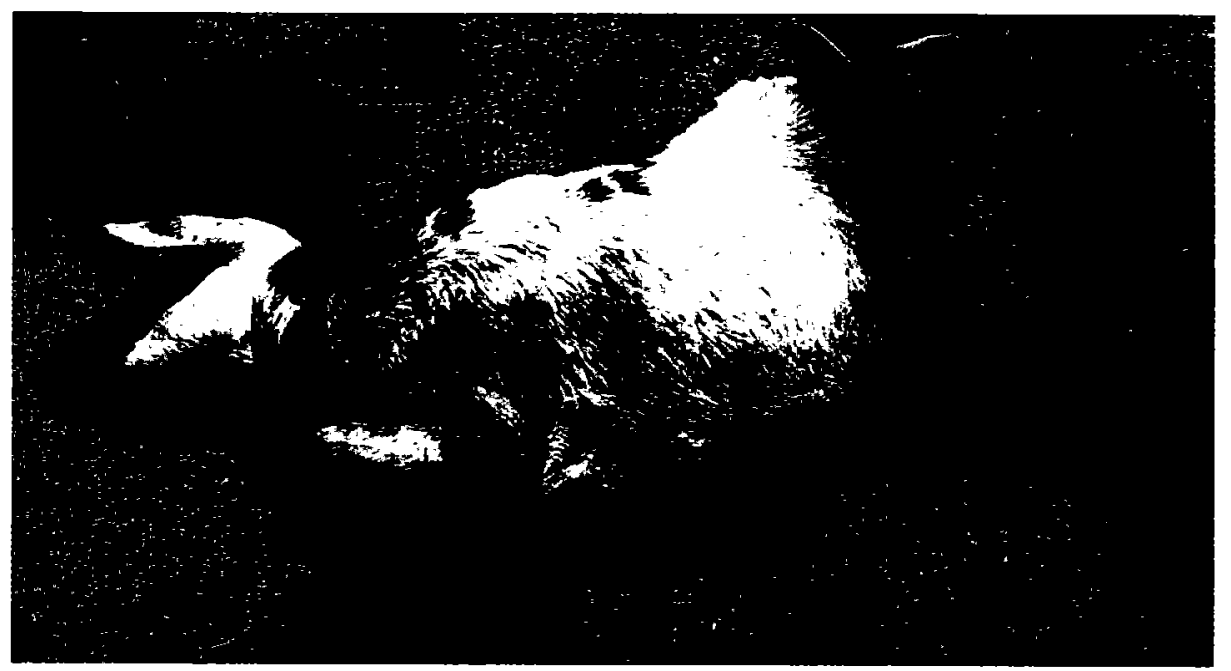

Phase terminale 
vie de décubitus, la mort survenant après un à quelques jours d'évolution (8). Pendant la phase ataxique, les animaux tremblent, présentent des symptômes de déséquilibre et se tiennent souvent sur les genoux. En phase de décutibus, on observe des crises de convulsion et de nystagmus. L'amaurose n'est qu'inconstamment mise en évidence.

\section{Diagnostic de laboratoire}

Les analyses parasitologiques, bactériologiques et virologiques n'ont mis en évidence aucun agent à pouvoir pathogène nerveux. Sur le plan hématologique, les modifications sont faibles et inconstantes. Les bilans biochimiques effectués sur 66 moutons malades montrent les résultats suivants : augmentation des CK (85 p. 100 des cas), des ASAT (75 p. 100) et, plus rarement, des LDH (30 p. 100) et des GGT (8 p. 100) ; peu de modifications des électrolytes et des sels minéraux, avec quelques augmentations de la phosphatémie (17 p. 100 des cas) et de la kaliémie ( 9 p. 100) ou diminution de la calcémie (15 p. 100) et des bicarbonates (11 p. 100). Les autres modifications constatées sont irrégulières et portent sur une augmentation de l'urée (15 p. 100 des cas), de la créatinine (3 p. 100), des triglycérides (22 p. 100) et du cholestérol $(35 \mathrm{p} .100)$ et une diminution des protéines totales (3 p. 100). Les taux d'acide lactique dépassent $2 \mathrm{mmol} / \mathrm{l}$ dans 95 p. 100 des cas (valeur extrême $12,8 \mathrm{mmol} / \mathrm{l}$ ) et ceux de l'acide pyruvique dépassent $100 \mu \mathrm{mol} / /$ dans 48 p. 100 des cas (valeur extrême $160 \mu \mathrm{mol} / \mathrm{l}$ ). Les résultats des autres dosages sont restés dans les normes (ALP, bilirubine totale et conjuguée, acide urique, sodium, chlorures et magnésium).

Les lésions histologiques du système nerveux central sont présentes dans 74 p. 100 des cas examinés (28 sur 38 ). Il s'agit de lésions de polioencéphalomalacie en foyers multiples, caractérisées par de l'oedème ou de la spongiose de la substance grise, associés dans les cas les plus évolués à des images de tuméfaction, de dégénérescence ou de nécrose des corps cellulaires des neurones situés dans la lésion. Ces lésions se localisent préférentiellement dans les noyaux du tronc cérébral et du bulbe, avec une atteinte constante et généralement symétrique des tubercules quadrijumeaux inférieurs. Les lésions du cortex sont inconstantes et plus discrètes : les foyers sont souvent délimités dans les régions corticales profondes et se traduisent par un aspect spongieux, discrètement vacuolaire, associé à des neurones rétractés ou d'aspect pycnotique. Les lésions du cervelet et de la moelle cervicale sont exceptionnelles.

\section{Traitement}

Sur les 72 moutons traités à des stades variés de la maladie, 56 ont guéri, soit 78 p. 100 des cas. Ces résultats sont variables selon la gravité ou l'ancienneté des symptômes :
- phase ataxique : 48 cas de guérison sur 49 traitements, soit 98 p. 100 de réussite ;

- phase de décubitus : 8 cas de guérison sur 23 traitements, soit 65 p. 100 de réussite.

\section{DISCUSSION}

\section{Assimilation du "syndrome nerveux ovin" à la nécrose du cortex cérébral (NCC)}

Le "syndrome nerveux" des ovins peut être assimilé à la nécrose du cortex cérébral, sur la base des éléments suivants :

- similitude parfaite des syndromes observés avec ceux décrits, dans la littérature pour la NCC $(2,4,7,10,11$, $14,20,21,22)$;

- présence de lésions de polioencéphalomalacie identiques à celles de la NCC $(14,15,16,21,22)$;

- efficacité remarquable du traitement exclusif avec le chlorhydrate de thiamine, aux doses élevées préconisées pour la NCC $(2,4,10,11,21,22)$.

Bien que parfois suspectée, la NCC n'avait pu être démontrée avant 1990 , sans doute parce que les analyses histologiques avaient été relativement peu nombreuses et n'avaient pas toujours porté sur la totalité du système nerveux central.

L'inconstance du traitement à la thiamine n'était, par ailleurs, pas bien argumentée. En effet, on peut constater que, le plus souvent, le traitement avec la vitamine B1 avait consisté en l'administration de cocktails vitaminiques polyvalents : la dose de thiamine injectée n'était donc en général pas suffisante pour pouvoir démontrer son efficacité. De plus, de nombreux malades furent traités à un stade trop avancé et donc incurable de la maladie (voir plus loin).

La maladie nerveuse relatée dans d'autres pays et dénommée "syndrome ataxie - parésie du mouton" au Ghana (3) ou "syndrome paraplégique du mouton de Casamance" au Sénégal (17), semble être un syndrome identique à celui décrit en Côte-d'lvoire. Mais l'absence, dans le syndrome nerveux ovin de Côte-d'lvoire, des lésions de la substance grise de la moelle épinière mises en évidence au Ghana reste pour le moment inexpliquée.

\section{Circonstances d'apparition et étiopathogénie}

Le syndrome nerveux apparaît, en Côte-d'Ivoire, dans des conditions très différentes de celles de la NCC dans les pays développés. En effet, la NCC est, classiquement, une maladie des jeunes ruminants à l'engrais, ali- 


\section{J. Domenech M. Wyers J.-P. Braun P. Formenty}

TABLEAU I Maladie à symptomatologie nerveuse (liste non exhaustive).

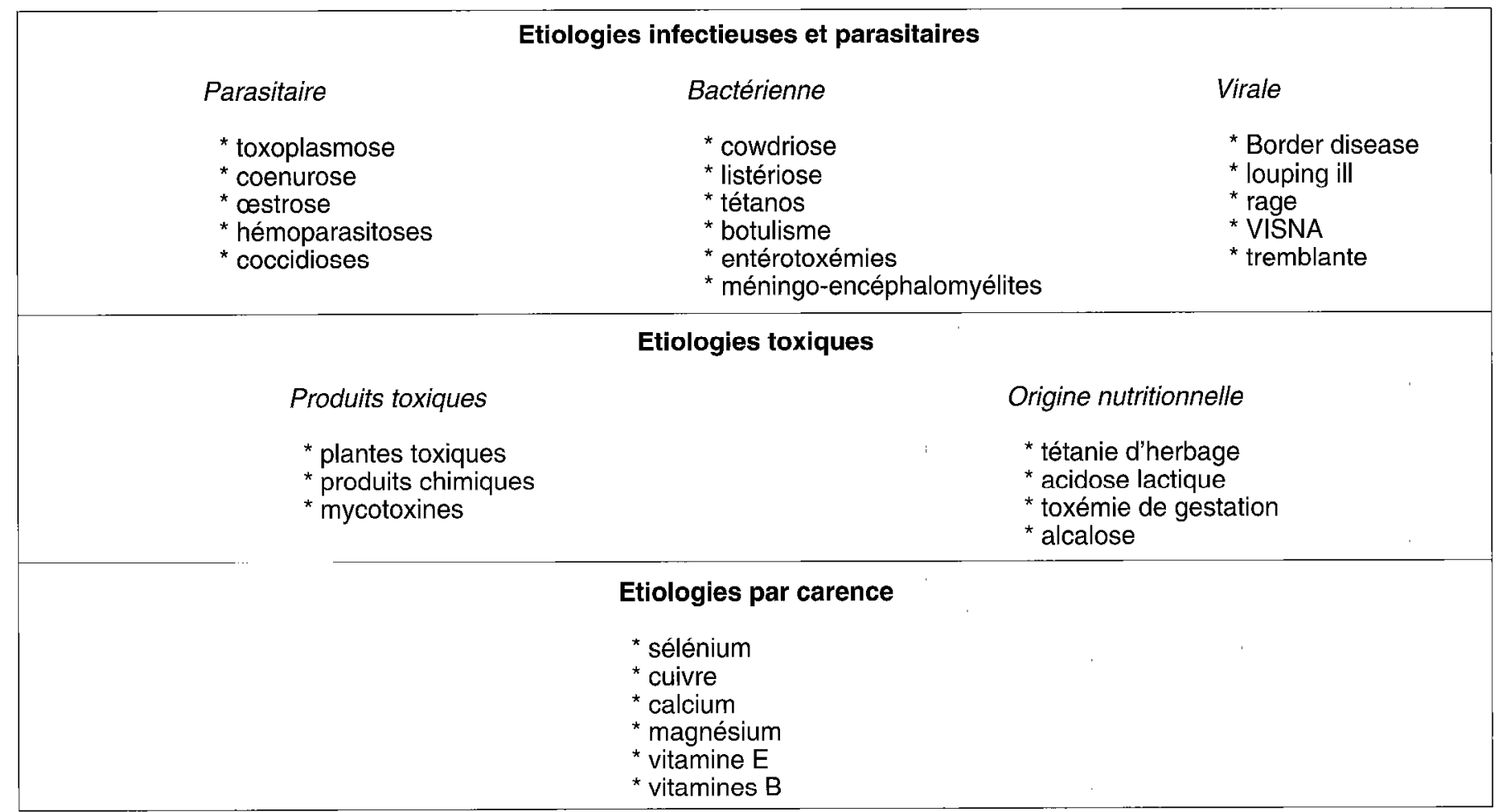

mentés de façon très intensive et subissant un déséquilibre nutritionnel brutal, dans le sens d'une augmentation de l'énergie et d'une diminution du taux des fibres $(1,9$, 21,22 ). La flore du rumen va alors se modifier et, soit ne plus produire la thiamine nécessaire à l'animal, soit produire une thiaminase ou un analogue structural de la thiamine $(4,9,10,21,22)$. Or, en Côte-d'lvoire, la maladie survient le plus souvent en saison sèche, lorsque le pâturage devient très sec et rare et que la complémentation alimentaire reste insuffisante.

Le syndrome nerveux est une conséquence directe d'un appauvrissement brutal et important de la ration alimentaire, dans des troupeaux d'une certaine taille et constitués de moutons améliorés sur le plan génétique, ayant donc des besoins plus élevés. Les animaux en divagation dans les villages, et qui ont des besoins moindres, parviennent, du fait de leur faible nombre, à glaner leur nourriture et sont épargnés par la maladie. L'étiopathogénie du syndrome nerveux reste donc assez mystérieuse et il est nécessaire de poursuivre un programme de recherches afin d'élucider les causes de cette carence en thiamine.

\section{Diagnostic du "syndrome nerveux" des ovins}

Si les symptômes sont assez évocateurs de la NCC, le diagnostic différentiel reste néanmoins à faire avec de nombreuses autres maladies à dominante nerveuse. Le tableau I présente une liste, non exhaustive, des affections parasitaires, bactériennes, virales, toxiques ou carentielles auxquelles il faut penser lorsqu'on est en présence de symptômes nerveux. II sera donc souvent indispensable d'effectuer un diagnostic biologique de confirmation. L'efficacité des injections de vitamine B1 sur l'animal malade représente un bon moyen mais il eut été intéressant de disposer d'une méthode de laboratoire applicable à l'animal vivant. Or, de toutes les études biochimiques réalisées, on n'a pu tirer aucun test qui soit à la fois spécifique, régulièrement positif et facile à utiliser.

Les CK sont certes augmentées dans la majorité des cas (85 p. 100) mais elles ne sont pas spécifiques de la lyse des cellules nerveuses, puisqu'elles sont également libérées lorsqu'il y a lyse des cellules musculaires (ce qui du reste est le cas lorsqu'on a, durant l'évolution du syndrome nerveux, des tremblements et convulsions musculaires). Les ASAT, élevées dans 75 p. 100 des cas, ne sont pas non plus suffisamment spécifiques pour assurer le diagnostic de NCC.

Les dosages de l'acide lactique et pyruvique devraient être plus intéressants, mais les conditions techniques de mise en eouvre (déprotéinisation et centrifugation du sang immédiates) et l'interférence du moindre effort musculaire, qui entraîne une augmentation des taux d'acide lactique et pyruvique (5), rendent ces dosages très délicats. De plus, outre sa mise au repos absolu, le malade devrait être à jeun, deux conditions impossibles à obtenir. 
EPIDEMIOLOGIE DESCRIPTIVE

Sur l'animal mort, le diagnostic histologique reste le plus sûr, mais encore faut-il prélever la totalité du système nerveux central et le plonger sans délai dans le formol à 10 p. 100 . Faute de quoi les artéfacts seront nombreux et difficiles à distinguer des lésions de polioencéphalomalacie. Par ailleurs, l'intégrité anatomique doit être soigneusement respectée afin que les tubercules quadrijumeaux, dans lesquels les lésions siègent de façon presque constante, puissent être facilement reconnus. Enfin, en cas de sacrifice en début de maladie, les lésions seront souvent discrètes et difficiles ou impossibles à déceler.

En conclusion, on voit que le diagnostic de confirmation reposera, pour l'animal vivant, sur le test d'efficacité du traitement avec la thiamine et, pour l'animal mort, sur I'histologie. Ajoutons cependant que la mesure de l'activité transcétolasique des globules rouges et le dosage de la vitamine B1 dans le foie et le cerveau (non pratiqués dans cette étude) sont envisageables si on dispose d'un laboratoire compétent pour effectuer ces analyses.

\section{Efficacité du traitement}

Le traitement avec le chlorhydrate de thiamine par voie intraveineuse et/ou sous-cutanée à forte dose $(400 \mathrm{mg}$ par jour), durant plusieurs jours, s'avère très efficace au point, ainsi qu'il a été dit plus haut, de constituer un élément déterminant du diagnostic de la maladie.

Le coût de ce traitement varie de 50 F CFA (1 FF) par animal, si on compte le seul prix du chlorhydrate de thiamine base (hors taxe, coût du travail de préparation de la solution et des flaconnages non compris) à 500 à $600 \mathrm{~F}$ CFA (10 à 12 FF) ${ }^{1}$ si on prend le prix TTC Abidjan d'un produit commercial à base de vitamine $\mathrm{B} 1^{2}$

DOMENECH (J.), WYERS (M.), BRAUN (J.-P.), FORMENTY (P.) The ovine nervous syndrome in Côte-d'Ivoire. I. An Epidemiological and clinical study, diagnostic methods and treatment. Revue Élev. Méd. vét. Pays trop., 1993, 46 (3): 423-430

The ovine nervous syndrome in Côte-d'Ivoire is similar to the cerebrocortical necrosis (CCN) due to vitamin B1 deficiency. All classical symptoms of CCN were observed (locomotor ataxia with subsequen paralysis) and histological evidence for polioencephalomalacia was given. However, the circumstances for occurrence of the disease are very different in the two cases, i.e. $\mathrm{CCN}$ is a disease encountered in young fattening ruminants in developed countries while the ovine nervous syndrome is mainly observed in Côte-d'Ivoire during the dry season when pastures become sparse and dry and when the feed supply is insufficient. Thus, the main cause, which is rather univocal, is a sudden decrease in the nutritive value of the diet, but the accurate etiopathogenesis of the disease has not yet been determined. In a flock where $10.30 \%$ of the animals are ill, the mortality may reach 80-90\%. No classical biochemical assays were specific enough to establish a precise diagnosis of the nervous syndrome. However, it should be pointed out that the CK (creatinine kinase) values very regularly rose and that the ASAT (aspartate aminotransferase) values were high in $75 \%$ of the cases. In the present African field conditions, the precise diagnosis is based on the efficiency of the vitamin B1 treatment and, for the dead animals, on the histological analysis of the brain.

Key words : Sheep - Nervous system disease - Cerebrocortical necrosis Poliencephalomalacia - Epidemiology - Diagnosis - Therapeutics - Histology - Vitamin deficiency - Thiamine - Côte-d'Ivoire.

\section{CONCLUSION}

Le syndrome nerveux des ovins en Côte-d'lvoire est une maladie due à une carence en vitamine B1. Elle se présente avec des caractéristiques cliniques et des lésions histologiques en tous points similaires à celles de la nécrose du cortex cérébral (NCC). De plus, le traitement avec de fortes doses de thiamine s'avère aussi efficace dans le syndrome nerveux ovin que dans la NCC. En revanche, les circonstances d'apparition du syndrome sont très différentes de celles décrites pour la NCC dans les pays développés. II y a donc un problème étiopathogénique à élucider. On sait que l'origine de la maladie est nutritionnelle et que l'utilisation d'une alimentation adaptée empêche souvent le syndrome nerveux de s'installer ou de trop se développer. Mais compte tenu des erreurs trop souvent commises par les éleveurs, il serait intéressant de disposer d'un schéma prophylactique efficace. Pour ce faire, des essais d'injection de thiamine ont été effectués, qui feront l'objet de la deuxième partie de l'exposé sur le syndrome nerveux des ovins. Par ailleurs, un programme de recherches sur cette maladie est en cours, afin de comprendre pourquoi et comment s'installe la carence en vitamine $\mathrm{B} 1^{3}$.

1. Les coûts en F CFA ne prennent pas en compte la récente dévaluation de celui-ci et sont à réactualiser en conséquence.

2. Corébral ${ }^{\oplus}$ (vétoquinol), Ultra $B$ (rigaux), Thiavit (CSI IVFC)...

3. Pour les remerciements et la bibliographie, se reporter à la fin de la deuxième partie de l'article, p. 519.

DOMENECH (J.), WYERS (M.), BRAUN (J.-P.), FORMENTY (P.). El síndrome nervioso de los ovinos en Costa de Marfil. I. Estudio epidemiológico y clínico, métodos de diagnóstico y tratamiento. Revue Élev. Méd. vét. Pays trop., 1993, 46 (3) : 423-430

EI "síndrome nervioso de los ovinos" en Costa de Marfil es similar a la necrosis del cortex cerebral ( $\mathrm{NCC}$ ), producida por una carencia de vitamina B1. Se han observado todos los síntomas clásicos de la NCC (ataxia locomotriz seguida de parálisis), así como las lesiones histológicas de polioencefalomalacia. Las condiciones de aparición de la enfermedad son, por el contrario, muy diferentes en los dos casos: si la NCC es una enfermedad de los jóvenes rumiantes de engorde en los países desarrollados, en Costa de Marfil, el síndrome nervioso de los ovinos se manifiesta esencialmente durante la estación seca, cuan. do los pastos están secos y son escazos y el complemento alimenticio es insuficiente. En forma general, la causa principal es supuestamente una degradación brutal del valor nutricional de la ración alimenticia, pero aún se desconoce la etio-patogenia exacta de la enfermedad. En un hato infectado, 10 a 30 p. 100 de los animales pueden enfermar, con una mortalidad de 80 a 90 p. 100. Desde el punto de vista diagnóstico, ninguna prueba bioquímica clásica es suficientemente específica como para ser utilizada para una confirmación formal del síndrome nervioso. Vale la pena señalar que las CK (creatinina kinasa) aumentan regularmente y que las ASAT (aspartato aminotransferasa) se encuentran elevadas en 75 p. 100 de los casos. EI diagnóstico formal debe centrarse, bajo las condiciones actuales de campo en Africa, sobre la eficiencia del tratamiento de la vitamina B1 y sobre el análisis histológico del cerebro del animal muerto.

Palabras claves : Ovino - Trastorno de sistema nervioso - Necrosis del cortex cerebral - Poliencefalomalacia - Epidemiología - Diagnóstico Terapéutica - Histología - Deficiencia de vitaminas - Tiamina - Costa de Marfil. 